\title{
Performance Evaluation of Weighted Fair Queuing System Using Matrix Geometric Method
}

\author{
Amina Al-Sawaai, Irfan Awan, and Rod Fretwell \\ Mobile Computing, Networks and Security Research Group \\ School of Informatics, University of Bradford, \\ Bradford, BD7 1DP, U.K. \\ \{a.s.m.al-sawaai,i.u.awan,r.j.fretwell\}@bradford.ac.uk
}

\begin{abstract}
This paper analyses a multiple class single server $\mathrm{M} / \mathrm{M} / 1 / \mathrm{K}$ queue with finite capacity under weighted fair queuing (WFQ) discipline. The Poisson process has been used to model the multiple classes of arrival streams. The service times have exponential distribution. We assume each class is assigned a virtual queue and incoming jobs enter the virtual queue related to their class and served in FIFO order.We model our system as a two dimensional Markov chain and use the matrix-geometric method to solve its stationary probabilities. This paper presents a matrix geometric solution to the $\mathrm{M} / \mathrm{M} / 1 / \mathrm{K}$ queue with finite buffer under (WFQ) service. In addition, the paper shows the state transition diagram of the Markov chain and presents the state balance equations, from which the stationary queue length distribution and other measures of interest can be obtained. Numerical experiments corroborating the theoretical results are also offered.
\end{abstract}

Keywords: Weighted Fair Queuing (WFQ), First Inter First Out (FIFO), Markov chain.

\section{Introduction}

Queue based on weighted fair queuing is a service policy in multiclass system. Consider a weighted service link that provides service for customers belonging to different classes. In WFQ, traffic classes are served on the fixed weight assigned to the related queue. The weight is determined according to the QoS parameters, such as service rate or delay. The WFQ [1] is a scheduling discipline usually applied to QoS enabled routers.

In this work, a two class single server $\mathrm{M} / \mathrm{M} / 1 / \mathrm{K}$ queue with a finite capacity under WFQ scheduling discipline is analyzed. The Poisson process is used to model two classes of arrival streams. The service times have exponential distribution. We assume each class is assigned a virtual queue and incoming jobs enter the virtual queue related to their class and served in FIFO order. The queue $i$ is served at rate $w_{i}$ for some $w_{i}>0$ when queue $1-i$ is not empty and at rate unity when that queue is empty. We model our system as a two dimensional Markov chain and use matrix geometric method to solve for its stationary probabilities. 
There were many solutions proposed to offer the solution for the WFQ system with two classes of customers on infinite buffer in [4] [5] and [6]. However, the class based with finite buffer is applied in a lot of computer and communication system and more realistic. The main aim of our work is to provide a solution to the WFQ system with a finite buffer. To the best of our knowledge to analytical and numerical solutions for such a system have not been in the literature.

The rest of this paper is organized as follows: An overview of related work is shown in Section 2. The queueing model of $\mathrm{M} / \mathrm{M} / 1 / \mathrm{K}$ queue with $\mathrm{WFQ}$ is described in Section 3. Section 4, offers a matrix geometric solution for $M / M / 1 / K$ queue under WFQ discipline, while section 5 presents and explains the numerical results of the model, followed by the conclusions and future work in Section 6 .

\section{Related Work}

The WFQ scheduling discipline is an important method for providing bounded delay, bounded throughput and fairness among traffic flows [2], [3]. The subject of WFQ has been investigated by many authors. Models similar to our WFQ system with two classes of customers have been analyzed for Poisson arrivals and exponential service times but with infinite buffers [4] and [5]. In [4], a two class system with two queues is considered; however the WFQ system is approximated with a two server, two queue systems and a numerical solution is provided. In [5], the same problem in [4] is considered; however an analytical solution for the system is given.

In [6], the authors provide an analytical solution for a WFQ system with a long range dependent traffic input with an infinite buffer and prove that analytical results provide an accurate estimation of queue length distribution and can be helpful in choices of WFQ weights. The work described in [7], defines an analytical solution of a WFQ system with two classes of customers with exponential service times in an unsteady state with an infinite buffer. The authors introduce an analytical model for the system and derive the exact expression of the tail of the probability distribution of the numbers of customers.

In [8], an analytical solution for the WFQ system with more than two queues and time connected variable service rates with an infinite buffer is provided. In addition a different design proposal for a dynamic WFQ scheduler is analyzed to provide quality of service guarantees.

This paper, we analyses the WFQ system with two classes of customers with a finite buffer in steady state and studies the effect of weights on the system mean a queue length, throughput and mean response time. To the best of our knowledge, the analysis of a WFQ system with finite buffer has not been proposed in the literature.

\section{The WFQ System Model}

Throughout this paper, we consider the same model in [4]; however instead of an infinite buffer, we use a finite buffer with size $\mathrm{K}$. The maximum number of customers who can be in the system at any time is $\mathrm{K}$ and any additional arriving customers will be refused entry to the system and will depart immediately without service. 


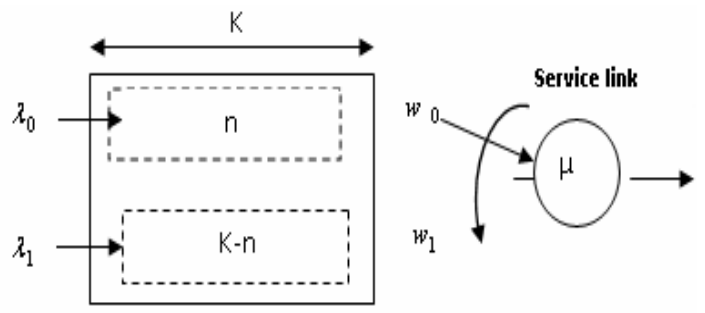

Fig. 1. The Weighted Fair Queuing System

For our WFQ system, we assume two classes of jobs; Jobs of class 1 and class 2 arrive according to a Poisson process with rate $\lambda_{i}, i=0,1$ and require exponential service times with mean $1 / \mu \mathrm{i}, i=0,1$. Each class is assigned a virtual queue and arriving jobs enter the virtual queue related to their class and are served in FIFO order.

The queue $i$ is served at rate $w_{i}$ for some $w_{i}>0$ when queue $1-i$ is not empty and at rate unity when that queue is empty. The coefficients $w_{i}$ are such that $w_{0}+w_{1}=1$. The server is work conserving, i.e., it serves jobs only if at least one queue is not empty. Fig. 1 depicts the WFQ system.

Fig. 2, illustrates the state-transition-rate diagram of the WFQ system where each state denotes the number of customers in the system. A generalized Markov model can be described by a two dimensional Markov chain with state $(i, j)$, where $i$ and $j$ are the number of customers in class 1 and class 2 at each state, respectively. When the process is in state $(i, j)$, it can transfer to the state $(i+1, j),(i, j+1),(i-1, j)$ if $i>0,(i, j-1)$. The transition rate from state $(i, j)$ to $(i+1, j)$ where $(0 \leq i \leq K-1)$ is the arrival rate of class 1 , i.e. $\lambda_{0}$, of the Poisson process. A transition out of state $(i, j)$ to $(i, j+1)$ where $(0 \leq j \leq K-1)$ is the arrival rate of class 2 , i.e. $\lambda_{1}$, of the Poisson process. When no customers of class 1 are in the system, the transition rate from $(0, j)$ to $(0, j-1)$ is the service rate of class 2 , i.e. $\mu_{1}$. The change from state $(i, 0)$ to $(i-1,0)$ is the service rate of class 1 , i.e. $\mu_{0}$. However, the transition rate from state $(i, j)$ to $(i-1, j)$ is the service rate of class 1 multiplied by the weight of class 1 , i.e. $w_{0} \mu_{0}$. A transition from $(i, j)$ to $(i, j-1)$ is the service rate of class 2 multiplied by the weight of class 2 , i.e. $w_{1} \mu_{1}$.

The infinitesimal generator of this process is given by:

$$
\begin{aligned}
& Q_{(i, 0),(i-1,0)}=\mu_{0}, i \geq 1 \\
& Q_{(i, j),(i-1, j)}=w_{0} \mu_{0} \\
& Q_{(i, j),(i, j-1)}=w_{1} \mu_{1}, i \geq 1, j \geq 1 \\
& Q_{(i, j),(i+1, j)}=\lambda_{0}
\end{aligned}
$$

and 


$$
Q_{(i, j),(i, j+1)}=\lambda_{1}, i \geq 0, j \geq 0
$$

The generator matrix $Q$ of the Markov chain is given by

$$
Q=\left(\begin{array}{ccccccc}
B_{0} & A_{1} & & & & \\
C_{0} & B_{1} & A_{2} & & & \\
& C_{1} & B_{2} & A_{3} & & \\
& & \ddots & \ddots & \ddots & \\
& & C_{n-2} & B_{n-1} & A_{n} \\
& & & & C_{n-1} & B_{n} \\
& & & & &
\end{array}\right)
$$

Where

$$
\begin{gathered}
C_{n}=\left(\begin{array}{ll}
\mu_{1} & \\
w_{0} \mu_{0} & w_{1} \mu_{1} \\
& w_{0} \mu_{0} \quad w_{1} \mu_{1}
\end{array}\right. \\
B_{n-1}=\left(\begin{array}{r}
-\left(\lambda+\mu_{1}\right) \\
-(\lambda+w \mu) \\
-(\lambda+w \mu)
\end{array}\right.
\end{gathered}
$$

In addition

$$
\lambda=\lambda_{0}+\lambda_{1}
$$

and

$$
w \mu=w_{0} \mu_{0}+w_{1} \mu_{1}
$$

The block entries $B_{0}, B_{1}, \ldots, B_{n}$ are square matrices, while the block entries $A_{1}, A_{2}, \ldots, A_{n}$ and $C_{1}, C_{2}, \ldots, C_{n}$ are rectangular matrices. The Markov chain with generator matrix $Q$ is irreducible; the matrices along the diagonal are non-singular. Below is the state equilibrium equations for all of the states of the Markov model of Fig. 2. 


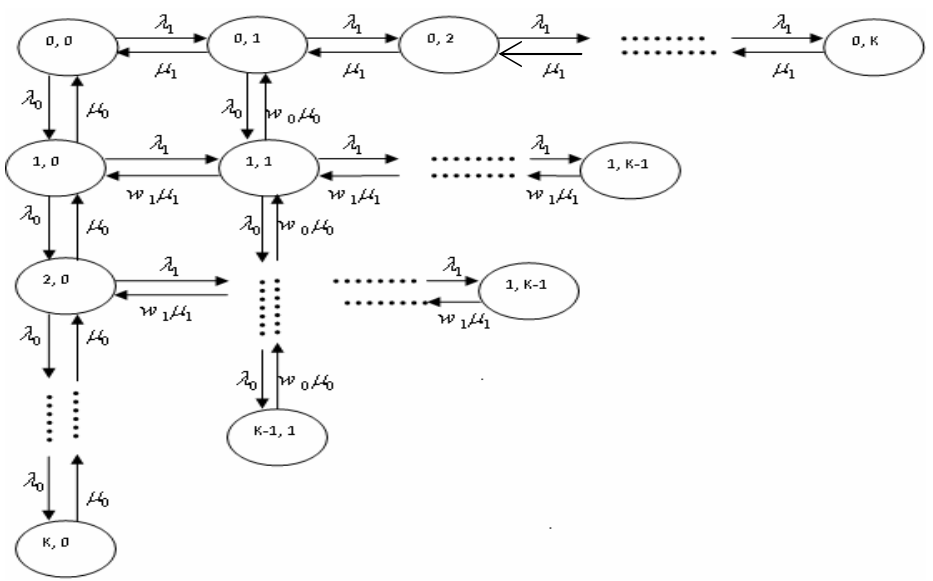

Fig. 2. The state transition diagram for an $M / M / 1 / K$ queue under WFQ scheduling discipline

\section{Matrix Geometric Solution:}

\subsection{State Equilibrium Equations}

The stationary probability vector $\underline{\pi}$ for $Q$ is generally partitioned as $\underline{\pi}=\left[\pi_{0}, \pi_{1}, \pi_{2}, \ldots \pi_{N}\right]$. Solving $\underline{\pi} Q=0$ along with normalizing equation $\underline{\pi} e=1$, yields the following set of equations in matrix form:

$$
\begin{aligned}
& \pi_{0} B_{0}+\pi_{1} C_{0}=0 \\
& \pi_{0} A_{1}+\pi_{1} B_{1}+\pi_{2} C_{1}=0 \\
& \pi_{n-1} A_{n}+\pi_{n} B_{n}+\pi_{n+1} C_{n}=0,1<n<N-1 \\
& \pi_{N-2} A_{N-1}+\pi_{N-1} B_{N-1}+\pi_{N} C_{N-1}=0 \\
& \pi_{N-1} A_{N}+\pi_{N} B_{N}=0
\end{aligned}
$$

Considering the above state equilibrium equations, it can be assumed that between any two states there is "flow in, flow out" equilibrium without any effect on the other remaining neighboring states.

\subsection{Matrix Geometric Method}

Suppose there exists a matrix $R$ as

$$
\pi_{n}=\pi_{n-1} R \quad \forall n \geq 1 .
$$

then, we get by successive substitutions into the state equilibrium equations that

$$
\pi_{n}=\pi_{0} R^{n} \quad \forall n \geq 0 .
$$


A solution of the (12) is called a matrix geometric solution [9]. The explanation for solving a matrix geometric system is to state the matrix $R$, the rate matrix, which is discussed below.

\subsection{Computation of the Rate Matrices}

By a simple algebraic stage management of the state equilibrium equations $R_{n}$ 's is formed as follows:

From Equation (6) and we know that $B_{0}$ is non-singular and we obtain,

$$
\pi_{0} B_{0}=-\pi_{1} C_{0} \rightarrow \pi_{0}=-\pi_{1} C_{0} B_{0}^{-1} \rightarrow \pi_{0}=\pi_{1} R_{0}
$$

Equation (10) leads to the following expression for $\pi_{N}$ and $R_{N}$ where $B_{N}$ is required to be non-singular,

$$
\pi_{N} B_{N}=-\pi_{N-1} A_{N} \rightarrow \pi_{N}=-\pi_{N-1} A_{N} B_{N}^{-1}
$$

Equation (9) leads to the following expression of $\pi_{N-1}$ and $R_{N-1}$

$$
\begin{aligned}
& \pi_{N-1} B_{N-1}+\pi_{N-1} R_{N} C_{N-1}=-\pi_{N-2} A_{N-1} \\
& \pi_{N-1}\left(B_{N-1}+R_{N} C_{N-1}\right)=-\pi_{N-2} A_{N-1} \\
& \pi_{N-1}=-\pi_{N-2} A_{N-1}\left(B_{N-1}+R_{N} C_{N-1}\right)^{-1} \\
& \pi_{N-1}=\pi_{N-2} R_{N-1}
\end{aligned}
$$

\begin{tabular}{|c|}
\hline Algorithm 1. Calculate $R_{n}$ \\
\hline $1: R_{N} \leftarrow-A_{N} B_{N}^{-1}$ \\
\hline $2:$ if $n \geq 1$ then \\
\hline 3: $\quad$ for $j=N-1 \rightarrow 1$ do \\
\hline 4: $\quad R_{j} \leftarrow-A_{j}\left(B_{j}+R_{j+1} C_{j}\right)^{-1}$ \\
\hline 5: $\quad$ end for \\
\hline 6: $\quad$ return $R_{n} \leftarrow-A_{n}\left(B_{n}+R_{n+1} C_{n}\right)^{-1}$ \\
\hline $7:$ end if \\
\hline 8: if $n==0$ then \\
\hline 9: $\quad$ return $R_{0} \leftarrow-C_{0} B_{0}^{-1}$ \\
\hline 10: end if \\
\hline
\end{tabular}

Finally from equation (8) we get a general relation between $\pi_{n-1}, \pi_{n}$ and $R_{n}$,

$$
\begin{aligned}
& \pi_{n}=-\pi_{n-1} A_{n}\left(B_{n}+R_{n+1} C_{n}\right)^{-1} \\
& \pi_{n}=\pi_{n-1} R_{n} \quad 2 \leq n<N-1
\end{aligned}
$$

$R_{n}$ can be calculated from Algorithm 1 . 


\subsection{Stationary Probabilities}

Theorem 1. For any QBD process with a finite state space, having an infinitesimal generator matrix given by Equation $Q$, the stationary probabilities are given in matrix-geometric form by

$$
\underline{\pi}_{n}=\underline{\pi}_{1} R_{n}^{*}
$$

Where $R_{n}^{*}=\prod_{j=2}^{N} R_{j}$ and $R_{j}$ is computed using Algorithm 1 .

Proof. The system of linear equations is solved for $\underline{\pi}_{1}$, and from equation (14), $\underline{\pi}_{n}$ is obtained as:

$$
\begin{aligned}
\underline{\pi}_{n}= & \underline{\pi}_{n-1} R_{n} \\
= & \underline{\pi}_{n-2} R_{n-1} R_{n} \\
& \vdots \\
= & \underline{\pi}_{1} R_{2} \ldots R_{n-2} R_{n-1} R_{n} \\
= & \underline{\pi}_{1} \prod_{j=2}^{n} R_{j}=\underline{\pi}_{1} R_{n}^{*}
\end{aligned}
$$

Solving Equation (6) and (7) for $\underline{\pi}_{1}$ and use $\underline{\pi}_{2}=\underline{\pi}_{1} R_{2}$ leads to,

$$
\underline{\pi}_{1}\left(R_{0} A_{1}+B_{1}+R_{2} C_{1}\right)=0
$$

Thus, after an exchange and mathematical manipulation, Equation (18) follows from normalizing condition $\sum_{n=0}^{N} \pi_{n} e=1$ and equation (6).

$$
\underline{\pi}_{1}\left(R_{0} e+\sum_{n=1}^{N} R_{n}^{*} e\right)=1
$$

It is worth noting that Theorem 1 giving the structure of the vector $\pi$ and that of the vector $\pi_{1}$ still needs to be determined. The vector $\pi_{1}$ could be computed from either one of the equations

$$
\begin{aligned}
& \underline{\pi}_{1}\left(R_{0} A_{1}+B_{1}+R_{2} C_{1}\right)=0 \\
& \underline{\pi}_{1}\left(R_{n-1}^{*} A_{n}+R_{n}^{*} B_{n}+R_{n+1}^{*} C_{n}=0, \quad 1<n<N-1\right. \\
& \underline{\pi}_{1}\left(R_{N-1}^{*} A_{N}+R_{N}^{*} B_{N}\right)=0
\end{aligned}
$$

and

$$
\underline{\pi}_{1}\left(R_{0} e+\sum_{n=1}^{N} R_{n}^{*} e\right)=1
$$




\begin{tabular}{l}
\hline Algorithm 2. Calculate $\underline{\pi}$ \\
\hline 1: for $j=2 \rightarrow n$ do \\
2: $\underline{\pi}_{j} \leftarrow \underline{\pi}_{1} \prod_{j=2}^{n} R_{j}$ \\
3: end for
\end{tabular}

Algorithm 2 is used to compute the stationary probabilities $\underline{\pi}$.

\section{Performance Analyses}

In this section, we present the results of the numerical calculations for an $\mathrm{M} / \mathrm{M} / 1 / \mathrm{K}$ queue under WFQ using (15) with different values for $\lambda_{0}, \lambda_{1}, \mu_{0}$ and $\mu_{1}$. The maximum buffer size for the system is 50 ; hence this model has 1326 states. The following is the numerical evaluation of the analytical model results for some performance measurements based on and derived from the Markov chain described in the previous section.

\subsection{Mean Queue Length}

The mean queue length $L$ is calculated from the model as follows:

$$
L=\sum_{i}^{K} i \pi(i)
$$

Where $\pi(i)=\pi(0), \pi(1), \ldots, \pi(K)$.

The following equations are derived from (22) to find the mean queue length for class $1, L_{0}$ and class $2, L_{1}$, respectively:

$$
\begin{aligned}
& L_{0}=\sum_{i=0}^{K} \sum_{j=0}^{K-i} i \pi(i, j) \\
& L_{1}=\sum_{j=0}^{K} \sum_{i=0}^{K-j} j \pi(i, j)
\end{aligned}
$$

where $\pi(i, j)$ is the steady state probability at state $(i, j)$.

Fig. 3 depicts the mean queue length for class 1 and class 2 for different arrival rates and with $w_{0}=w_{1}=0.5$. We choose $\mu_{0}=\mu_{1}=1$ to only examine the effect of the queue weights. Using these parameters, the WFQ system reduces to two $\mathrm{M} / \mathrm{M} / 1 / \mathrm{K}$ systems. It can be seen from Fig. 3 that the mean queue length for both classes increases with the increase of the arriving traffic until $\lambda=1$ then $L_{0}=L_{1} \approx 24.5$. The total of $L_{0}$ and $L_{1}$ equals the buffer size: $\mathrm{K}=50 . L_{0}$ and $L_{1}$ have identical values for all arrival rates. 
In Fig. 4 , we choose $w_{0}=0.6$ and $w_{1}=0.4$ for different values of $\lambda$ when $\mu_{0}=2, \mu_{1}=1$. It indicates that the mean queue length for class 1 is lower than the mean queue length for class 2 , as expected. Class 1 is served faster than class 2 at rate $w_{0} \mu_{0}=1.2$. We find the same results for $L_{1}$, when $w_{0}=0.4, w_{1}=0.6$ and $\mu_{0}=1, \mu_{1}=2$.

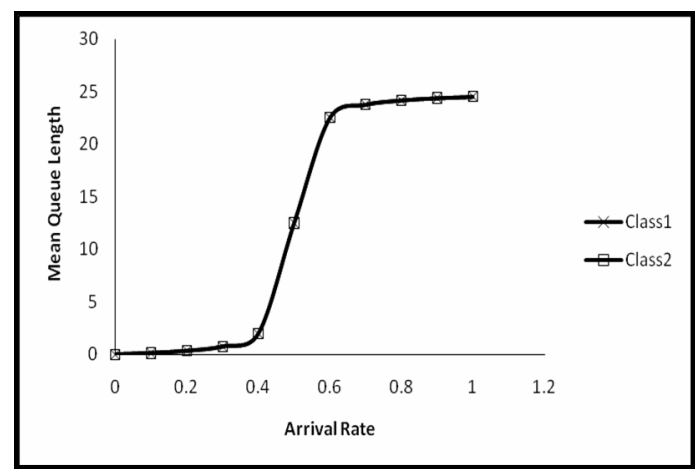

Fig. 3. Mean queue length, $w_{0}=w_{1}=0.5$ and $\mu_{0}=\mu_{1}=1, \lambda_{0}=\lambda_{1}$

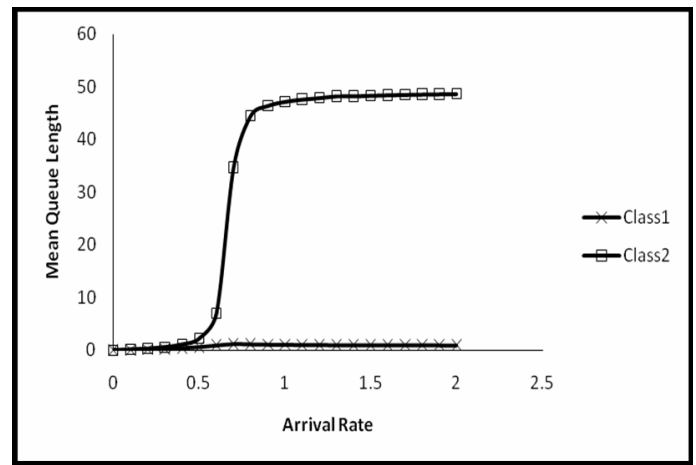

Fig. 4. Mean queue length, $w_{0}=0.6, w_{1}=0.4$ and $\mu_{0}=2,=\mu_{1}=1, \lambda_{0}>0, \lambda_{1}>0$

Fig. 5 depicts the mean queue length for different values of class1 with an arrival rate $\lambda_{0}>0$ and fixed arrival rate $\lambda_{1}=0.2$ with $w_{0}=0.6, w_{1}=0.4$. We make a note of that as $\lambda_{0}$ increases, and then $L_{0}$ increases until the mean queue length approaches to 50 and then it is stable. However, the mean queue length of class 2 increases and then decreases to zero. The $L_{1}$ is lower in this case compared to $L_{0}$. The reason why $L_{0}$ is higher than $L_{1}$ is the fact that class 1 is getting a higher service rate, $w_{0} \mu_{0}=1.2$ and its 
increasing arriving traffic causes class 2 to be refused entry to the system. This can be observed in Fig. 5.

\subsection{System Throughput}

The following equation is used to find the system throughput $(T$ ) for the $\mathrm{M} / \mathrm{M} / 1 / \mathrm{K}$ queue from the analytical model:

$$
T=\mu(1-\pi(0))
$$

- $\quad \mu$ is the output rate, when the server is busy .

- $\pi($ server is busy $)=(1-\pi($ server is idle $)=(1-\pi(0))$.

- when the server is idle, the output rate equals zero.

Equation (25) is used to derive the throughput ( $T$ ) for class $1, T_{0}$, and class $2, T_{1}$, respectively:

$$
\begin{gathered}
T_{0}=w_{0} \mu_{0}(1-\pi(0)) \\
T_{1}=w_{1} \mu_{1}(1-\pi(0))
\end{gathered}
$$

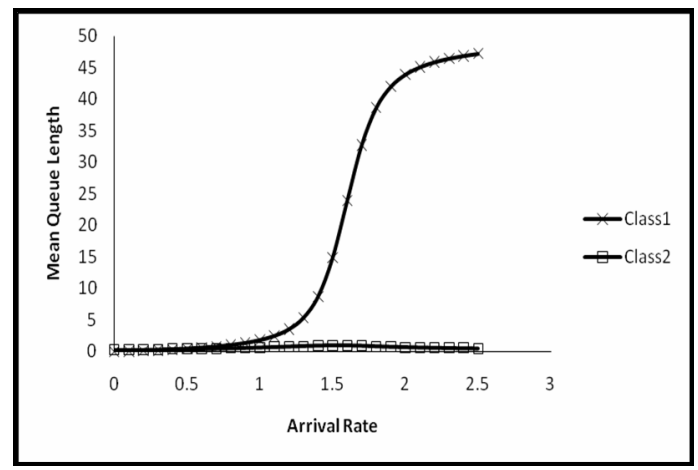

Fig. 5. Mean queue length, $w_{0}=0.6, w_{1}=0.4$ and $\mu_{0}=2, \mu_{1}=1, \lambda_{0}>0, \lambda_{1}=0.2$

Fig. 6 shows $T_{0}$ with $w_{0}=0.6$ and $T_{1}$ with $w_{1}=0.4$ for different arrival rates of class 1 and class $2, \lambda_{0}>0, \lambda_{1}>0$. It indicates that $T_{0}$ depends on the weight of class 1 and that it means $T_{0} \approx w_{0} \mu_{0}$ and $T_{1} \approx w_{1} \mu_{1}$. The system throughput will not exceed the services rate (in this example: $w_{0} \mu_{0}+w_{1} \mu_{1}=1.6$ ).

In Fig. 7, we obtain something like the results for $T_{0}$ with $w_{0}=0.6$ and $T_{1}$ with $w_{1}=0.4$ for different arrival rates of class 1 and class $2, \lambda_{0}>0, \lambda_{1}>0$. However, the graph will be stable with arrival rate $\lambda_{0}=\lambda_{1}=2.3$ for both classes. 


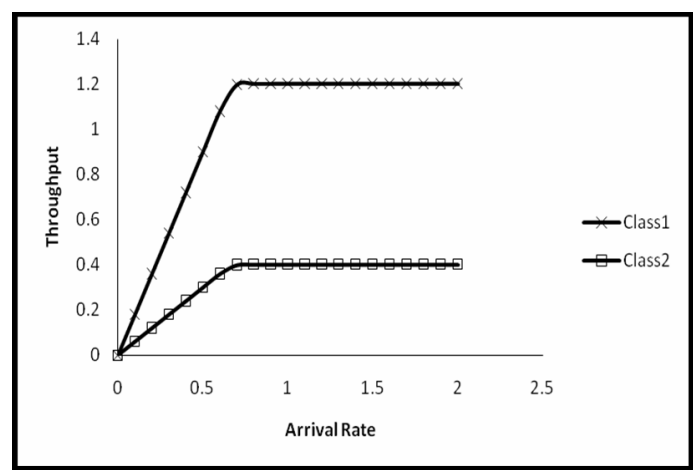

Fig. 6. Throughput, $w_{0}=0.6, w_{1}=0.4$ and $\mu_{0}=2, \mu_{1}=1, \lambda_{0}>0, \lambda_{1}>0$

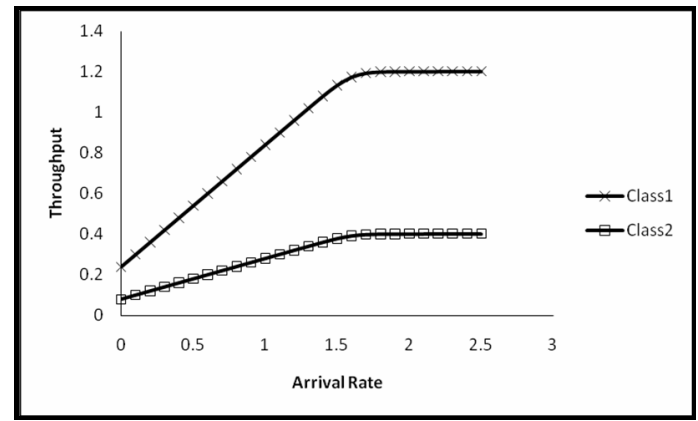

Fig. 7. Throughput, $w_{0}=0.6, w_{1}=0.4$ and $\mu_{0}=2, \mu_{1}=1, \lambda_{0}>0, \lambda_{1}=0.2$

\subsection{The Mean Response Time}

Using Little's law [10]:

$$
L=T S
$$

We can derive the mean response time for class $1, S_{0}$, and class $2, S_{1}$, from (28) respectively as:

$$
\begin{gathered}
S_{0}=L_{0} / T_{0} \\
S_{1}=L_{1} / T_{1}
\end{gathered}
$$

where $T_{0}$ and $T_{1}$ are measured system throughput when packet loss is considered ( $T_{\text {Effective }}$ ).

Fig. 7 shows the mean response time of class 1 and class 2 when $\mu_{0}=2, \mu_{1}=1$ and $w_{0}=0.6, w_{1}=0.4$. We can observe that $S_{1}$ is higher than $S_{0}$ and class 2 stays longer in the system. The reason why the mean response time has turned out to be higher for class 2 is the fact that it has a low weight. 


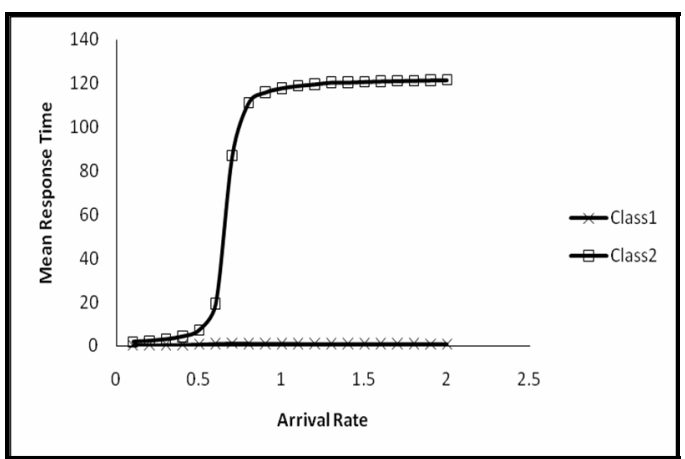

Fig. 8. Mean response time, $w_{0}=0.6, w_{1}=0.4$ and $\mu_{0}=2, \mu_{1}=1, \lambda_{0}>0, \lambda_{1}>0$

Fig. 8 depicts the mean response time for different values of class 1 with the arrival rate $\lambda_{0}>0$ and the fixed arrival rate $\lambda_{1}=0.2$ with $w_{0}=0.6, w_{1}=0.4$. We can calculate that as $\lambda_{0}$ increases, $S_{0}$ increases until the mean response time approximately equals 40 and then it is stable. However, the mean response time of class 2 increases until 2.4 and then decreases to zero. The reason why $S_{0}$ is a higher than $S_{1}$ is the fact class 1 is receiving different arrival rates and a higher service rate, $w_{0} \mu_{0}=1.2$.

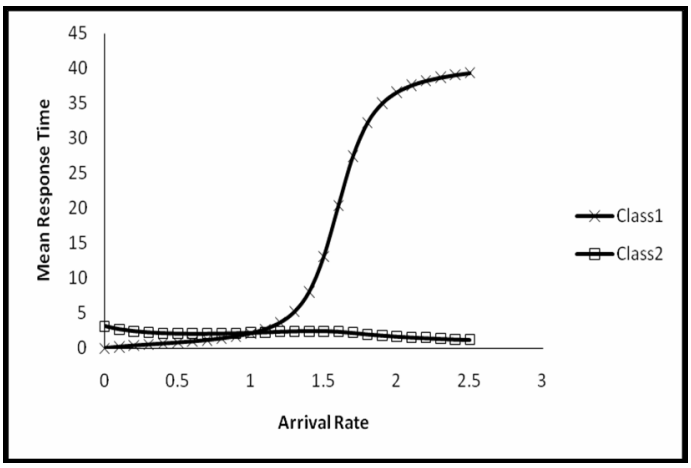

Fig. 9. Mean response time, $w_{0}=0.6, w_{1}=0.4$ and $\mu_{0}=2, \mu_{1}=1, \lambda_{0}>0, \lambda_{1}=0.2$

For all obtained performance measurements, the WFQ system behaved as expected. These numerical results confirm the validity of the analytical model.

\section{Conclusions and Future Work}

In this paper, we provide an analysis of the two class single server $\mathrm{M} / \mathrm{M} / \mathrm{1} / \mathrm{K}$ queue with a finite capacity under a weighted fair queuing scheduling discipline. The 
Poisson process has been used to model the multiple classes of arrival streams. The service times have exponential distribution. An analytical expression for the flow balanced equations has been derived using a Markov chain. Queue length distribution has been derived by solving these expressions. We derived a general expression for the steady state probabilities for any finite buffer with size K. In addition, we found the steady state probabilities $\pi_{i, j}$ for M/M/1/50 queue with WFQ as an example and we presented the numerical results. Future work will focus on deriving general equations for the performance measure for an $\mathrm{M} / \mathrm{M} / 1 / \mathrm{K}$ queue with a finite capacity under a weighted fair queuing (WFQ) with more than two classes. We will also extend it with more realistic traffic models.

\section{References}

1. Li, C., Tsao, S., Chen, M., Sun, Y., Huang, Y.: Proportional Delay Differentiation Service Based on Weighted Fair Queuing. In: Proceedings Ninth International Conference on Computer Communications and Networks, 2000, pp. 418-423 (2000)

2. Parkekh, A.K., Gallager, R.G.: A generalized processor sharing approach to flow control in integrated services networks: The single-node case. IEEE Trans. On Networking 1(3), 344-357 (1993)

3. Parekh, A.K., Gallager, R.G.: A generalized processor sharing approach to flow control in integrated services networks: The multiple node case. IEEE Trans. On Networking 2(2), 137-150 (1994)

4. Horváth, G., Telek, M.: Approximate analysis of two class WFQ systems. In: Workshop on Preformability Modeling of Computer and Communication Systems - PMCCS 2003, Arlington, IL, USA, September 2003, pp. 43-46 (2003)

5. Guillemin, F., Pinchon, D.: Analysis of the Weighted Fair Queuing System with Two Classes of Customers with Exponential Service Times. Journal of Applied Probability (2004)

6. Ashour, M., Le-Ngoc, T.: Performance of Weighted Fair Queuing System with Long Range Dependent Traffic Inputs. In: Electrical and Computer Engineering, pp. 1002-1005 (2005)

7. Davis, P.F., Rabinowitz, P.: Methods of numerical intergration, 2nd edn. Academic Press, London (1984)

8. Ashour, M., Le-Ngoc, T.: Performance of Weighted Fair Queuing System with Variable Service Rates. In: International Conference, pp. 51-57 (2006)

9. Daigle, J.: Queueing Theory for Telecommunications, 1st edn., pp. 138-147. AddisonWesley Longman (1991)

10. Adan, I., Resing, J.: Queueing Theory, Eindhoven University of Technology, Department of Mathematics and Computing Science, February 14 (2001) 